\title{
Energías renovables y el hidrógeno: un par prometedor en la transición energética de México
}

\author{
Renewable energies and hydrogen: a promising couple \\ in the energy transition of Mexico

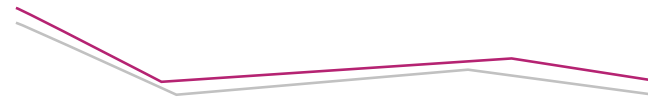 \\ Alejandra Cecilia Morales Ramos ${ }^{1}$, Marisela Pérez Figueroa ${ }^{2}$, \\ Jorge Raúl Pérez Gallardo3*, Sofía De León Almaraz ${ }^{4}$ \\ Morales Ramos, A. C. Pérez Figueroa, M. Pérez Gallardo, J. R. De León Almaraz, S. Energías \\ renovables y el hidrógeno: un par prometedor en la transición energética de México. Investigación \\ y Ciencia de la Universidad Autónoma de Aguascalientes. Número 70: 92-101, enero-abril 2017.
}

\section{RESUMEN}

Existen numerosas razones para considerar seriamente el uso de energías renovables (ER) en el portafolio de recursos utilizados en México: seguridad e independencia energética, reglamentación y compromisos ambientales, aprovechamiento de recursos nacionales, entre otros. México debe replantear su dependencia por combustibles fósiles como petróleo y gas natural en su transición energética. El hidrógeno es una opción que está siendo analizada por la comunidad científica internacional para acoplarse a las ER, ya que es un portador de energía que ofrece una solución a la intermitencia de las ER y puede servir para almacenar la energía. El hidrógeno ofrece grandes ventajas ambientales en su uso, y resulta una opción muy prometedora. Este estudio tiene como objetivo identificar el potencial que tienen las ER en México al ser utilizadas eficientemente para almacenar la energía por medio del hidrógeno. Se identifican el tipo, ubicación, capacidad y costo de las principales ER en México. Asimismo, se establece un escenario prospectivo de capacidad de almacenamiento mediante el acoplamiento de las $\mathrm{ER}-\mathrm{H}_{2}$ para cuatro

Palabras clave: energía renovable, hidrógeno, ambiente, prospectivas, vector energético.

Keywords: renewable energy, hydrogen, environment, prospective, energy carrier.

\footnotetext{
Recibido: 7 de abril de 2016, aceptado: 20 de octubre de 2016

Instituto Tecnológico Superior de Apatzingán, México.

Instituto Tecnológico Superior de Los Ríos, México.

Centro de Investigación en Matemáticas, A.C. (CIMAT-Conacyt)

unidad Aguascalientes, México.

$4 \quad$ Laboratorio de Ingeniería Química, Universidad de Toulouse, Francia.

* Autor para correspondencia: raul.perez@icimat.mx
}

periodos de tiempo (2015, 2020, 2025 y 2029). Las estimaciones muestran una capacidad de almacenamiento de hasta 71,135 GWh/año por medio del hidrógeno al año 2029. Los resultados de esta investigación servirán como referencia para realizar estudios más detallados para el diseño de cadenas de suministro para ER-H

\section{ABSTRACI}

There are many reasons to consider the use of renewable energies (RES) in the Mexican resources portfolio: energy security and autonomy, regulatory and environmental commitments, utilization of national resources, etc. Mexico must rethink its dependence on fossil fuels, such as oil and natural gas in their energy transition. Hydrogen is an option that is being considered by the international scientific community to be coupled to the RES because it is an energy carrier that offers a solution to the intermittency and can be used to store energy. Hydrogen provides significant environmental advantages in their use. This study aims to identify the potential of RES in Mexico to be used efficiently to store energy through hydrogen. First, the type, location, capacity and cost of major RES in Mexico are identified. With the above scenario a prospective storage capacity is calculated by coupling the RES- $\mathrm{H}_{2}$ for four time periods $(2015,2020$, 2025 and 2029). The results show a storage capacity of up to 71,135 GWh/year in 2029 through hydrogen. The results of this research will serve as a reference for more detailed design studies for supply chains to ERs- $\mathrm{H}_{2}$.

\section{INTRODUCCIÓN}

En la última década el interés por el uso eficiente de recursos y energía ha cobrado una gran relevancia. 
Existen presiones de orden nacional e internacional para poder desarrollar una economía sostenible que garantice el aseguramiento energético, el crecimiento económico y el cuidado al medio ambiente (Bhattacharya et al., 2016; Scholten y Bosman, 2016). A pesar de las tensiones por los combustibles fósiles y la creciente volatilidad de los precios del petróleo (Buyuksahin, 2012; Salameh, 2014; Kottasova, 2015), las energías fósiles seguirán siendo la base del desarrollo económico mundial. Sin embargo, se espera que durante las siguientes décadas las fuentes renovables adquieran mayor relevancia, y se conviertan en un elemento esencial para el desarrollo y bienestar futuros de la humanidad (International Energy Agency, 2014, 2015; Bhattacharya et al., 2016).

Además, hay una exigencia mayor de contar con herramientas de planeación indicativa, que coadyuve en la toma de decisiones en el mercado energético y permita evaluar con mayor precisión la disponibilidad y demanda de las distintas fuentes energéticas en el mediano plazo, lo que brinda certeza sobre los proyectos de infraestructura, así como elementos para la inversión por parte del sector privado (SENER, 2015b).

El presente trabajo tuvo como objetivo indagar el potencial que tendría el uso de energías renovables (ER) en nuestro país al maximizar su eficiencia e incrementar su penetración mediante el uso del $\mathrm{H}_{2}$ como vector energético para su almacenamiento y posterior utilización. En primer lugar, se ofrece un panorama general sobre el estado actual del sector energético nacional, así como de las distintas formas de generación energética mediante fuentes renovables. El propósito es determinar los principales tipos de ER existentes en el país, la capacidad instalada, la ubicación de las plantas generadoras y los costos de instalación para cada fuente de energía. A partir de la información recabada se estima el potencial de almacenamiento de energía mediante la producción de $\mathrm{H}_{2}$ a partir de ER siguiendo la metodología propuesta en la tesis de De León Almaraz (2014). Finalmente, se ofrece un panorama hasta el año 2029 del posible crecimiento de este sector y la importancia del uso del $\mathrm{H}_{2}$ como vector energético.

\section{Contexto energético mexicano}

La dependencia nacional al petróleo, el incremento de la demanda energética nacional, así como la incapacidad del Estado para invertir en obra tendiente a incrementar la capacidad existente ha constituido un problema muy serio para el país (Cadet, 2003).

En la década de los ochenta, el petróleo llegó a representar casi $65 \%$ del valor total de las exportaciones, mientras que en 2000 su participación disminuyó a $8.9 \%$ (CEFP, 2001). Hasta 2013, todos los procesos relacionados al manejo de hidrocarburos y electricidad estaban centralizados en Petróleos Mexicanos (PEMEX), Comisión Federal de Electricidad (CFE) y Luz y Fuerza del Centro (LFC).

A fines de 2013 se decidió implementar un nuevo modelo de contratación en la exploración y extracción de hidrocarburos al reformar la legislación vigente. Dicho modelo permite la inversión privada y la competencia en actividades de transporte y transformación industrial de hidrocarburos (SENER, 2015b). La reforma intenta cumplir con los compromisos ambientales que México ha suscrito.

\section{Compromisos ambientales y acuerdos internacio-} nales. En 2007, el Grupo Intergubernamental de Expertos sobre el Cambio Climático (IPCC: Intergovernmental Panel on Climate Change) presentó un reporte alarmante en el que se concluyó que al ritmo de crecimiento actual la temperatura del planeta aumentaría $6^{\circ} \mathrm{C}$ para el fin de siglo XXI (IPCC Core Writing Team et al., 2007). En 2009, la Agencia Internacional de Energía presentó diversos escenarios y estrategias de mitigación por país con el fin de limitar el aumento de la temperatura a $2^{\circ} \mathrm{C}$ en el año 2100 (International Energy Agency, 2009). Las recomendaciones hechas para México son establecer un mecanismo de mercado para incentivar la reducción de emisiones de $\mathrm{CO}_{2}$ al mínimo costo (sistema cap-andtrade) para los sectores de energía e industria, adoptar acuerdos internacionales para industria y transporte, y aplicar políticas nacionales de disminución de contaminantes (Energía y Sociedad, 2014).

En 2015, la Conferencia de la ONU sobre Cambio Climático (COP 21) en París buscó impulsar un ambicioso acuerdo internacional para una nueva era de desarrollo sostenible (United Nations, 2015). En la COP 21 el presidente Enrique Peña Nieto destacó la aprobación del impuesto al carbono para aquellos combustibles fósiles con emisiones superiores a las del gas natural, y el avance en la creación del mercado de carbono, enmarcados en la reforma energética recién aprobada. Asimismo, resaltó la meta impuesta 
de reducir para el año 2030 en $22 \%$ las emisiones de gases de efecto invernadero y en $51 \%$ las de carbono negro a nivel nacional (México News Network, 2015).

En la cumbre de líderes de América del Norte 2016, los presidentes de México, Canadá y Estados Unidos se comprometieron a alcanzar $50 \%$ de generación de electricidad limpia en 2025 (Presidencia de la República, 2016). Hablar de energía limpia no es sinónimo de energía renovable sino de la sustitución de algunas fuentes de energía por otras menos contaminantes; por ejemplo, pasar del uso de carbón al del gas natural para la producción de electricidad. Lo anterior hace más lenta la transición hacia el Uso de ER. Este panorama internacional vuelve necesaria una acelerada transición hacia energías no solo más limpias sino renovables en el balance energético nacional si se analizan los principales puntos de consumo y los usos de energía en México.

Situación actual. En el informe Participación de las Energías Renovables en la Generación de Electricidad en México 2014 (SENER, 2015a) se establecieron metas de capacidad y generación de energía. La Secretaría de Energía (SENER) y el congreso han fijado una participación máxima de $65 \%$ de combustibles fósiles en la generación de energía eléctrica para el año 2024, de 60\% en 2035 y de $50 \%$ en 2050 (SEGOB, 2008).

En años recientes, el gobierno promueve el uso de gas natural (GN) como una fuente alternativa de energía mediante inversiones en plantas de ciclo combinado y gasoductos (CEFP, 2001). El GN es el combustible de menor precio en el mercado y se ha constituido como un combustible de transición fundamental para el país (SENER, 2015b) debido a su bajo precio en ciertas regiones, mejores eficiencias en centrales de generación eléctrica y las ventajas en cuanto a emisiones en comparación con otros tipos de combustibles fósiles (CEFP, 2001; SENER, $2015 b, 2015 c)$. A pesar de que México es productor de GN, surge una alerta debido al considerable incremento en las importaciones de este producto (Cadet, 2003). Si bien el GN es una fuente más limpia para la atmósfera, al ser no renovable, puede ser superado en beneficios ambientales por otras fuentes de energía.

LaSENER, con la Comisión Federal de Electricidad (CFE) y la Comisión Reguladora de Energía (CRE), promueven el uso de energías limpias con una participación superior a 35\% para el año 2024 y a 43\% para 2030. Ante esto, México necesita un sector energético diversificado, en el que una canasta de fuentes de ER distintas sea un componente esencial para alcanzar la seguridad energética, mejorar la competitividad presente y lograr los ambiciosos objetivos ambientales que se ha planteado.

Acerca de la demanda energética, se encuentra que en 2013 el Balance Nacional de Energía (SENER, 2014a) indicó que el sector transporte consume $44.1 \%$ de la energía generada, seguido de la industria con $31.4 \%$ y $17.7 \%$ por los fragmentos residencial, comercial y público. El consumo energético por tipo de combustible muestra al uso de gasolina en primer lugar (29.8\%), seguido por la electricidad (17.1\%) y el diésel (16.0\%). La suma de gasolina y diésel resulta en $45.8 \%$ del consumo total, lo que demuestra la gran dependencia a los derivados del petróleo y alerta sobre las emisiones generadas por el uso de este tipo de carburantes en los vehículos de combustión interna. Entre el período comprendido desde 2004 hasta 2014 se incrementó el consumo nacional de energía eléctrica a una tasa promedio de $2.9 \%$ anual, para ubicarse en 244,673 GWh en 2014 (SENER, 2015d).

\section{$\mathrm{H}_{2}$ como vector energético}

El hidrógeno $\left(\mathrm{H}_{2}\right)$ es un vector energético que tiene grandes ventajas ambientales. Su característica principal es que, aunque no es en sí una fuente energética, puede tener diferentes orígenes, tanto renovables como no renovables. Es un combustible limpio, cuando se quema con aire las emisiones que produce son no contaminantes. Además de la combustión directa, se ha empezado a desarrollar una tecnología basada en pilas de combustibles en las que se transforma la energía química en energía eléctrica y vapor de agua.

De forma natural el hidrógeno se encuentra en cantidades mínimas, por ello se deben realizar ciertas transformaciones térmicas, químicas o electroquímicas para obtenerlo. Los métodos más comunes son el reformado de gas natural, la gasificación del carbón o de biomasa y la electrólisis de agua. De ellos, la electrólisis del agua resulta ser un proceso limpio si la energía necesaria para realizar el proceso es suministrada vía ER (ARIEMA, 2003a). El mayor porcentaje de la producción de $\mathrm{H}_{2}$ en México es obtenido por reformación catalítica de hidrocarburos, procesos electrolíticos y métodos en los que se genera hidrógeno como producto 


\section{IIVESTIGAGIÓn Y CUERCIA DE LA UחIVERSIDAD AUTÓNOMA DE RGUASCALIERTES}

secundario de otros procesos químicos (Galarza, 2008).

En el mundo existen iniciativas que toman al $\mathrm{H}_{2}$ como vector clave en la transición energética y de movilidad (Air liquide, 2008; Scandinavian Hydrogen Highway Partnership, 2011; NOW, 2012). Muchas de estas iniciativas ya se encuentran operando.

El hidrógeno coadyuva de forma importante a resolver los problemas de almacenamiento y transporte de energía que en el futuro provocará el uso masivo de ER. Durante el periodo de transición energética permitirá un uso más eficiente y menos contaminante de los combustibles fósiles; sin embargo, existen factores económicos y de seguridad pendientes por resolver para una utilización rentable del hidrógeno, ya que es difícil justificar el uso del hidrógeno exclusivamente desde una perspectiva de política climática. El principal problema que enfrenta es que debe ser producido por medio de otra fuente energética debido a que sólo puede encontrarse en forma de compuesto sobre la Tierra. Por tanto, sus ventajas ambientales dependerán de cómo es producido. Si se elabora a partir de fuentes fósiles, aumenta la seguridad del suministro, pero hace que las emisiones de $\mathrm{CO}_{2}$ sean elevadas (a menos que el $\mathrm{CO}_{2}$ sea capturado y almacenado). $\mathrm{Si}$ es creado mediante combustibles no fósiles (nuclear o renovable), se reducen las emisiones de $\mathrm{CO}_{2}$ pero el suministro estará condicionado por la cantidad de recurso no fósil disponible y que no sea empleado para la generación de electricidad (Ball and Wietschel, 2009).

Las energías renovables (ER) y el hidrógeno $\left(\mathbf{H}_{2}\right)$. La transición hacia el uso de ER en el futuro parece inevitable; sin embargo, con el uso de las mismas también surgen problemas asociados a la intermitencia (Fernández Ordóñez, 2010). Este tipo de energías presentan una gran dependencia climatológica y, por tanto, una alta variabilidad. Para superar esta barrera se puede almacenar la energía por medio de baterías o del uso del hidrógeno como vector energético. La capacidad limitada de las baterías hace al hidrógeno una opción muy prometedora para acoplarse al uso de las ER, a pesar de la existencia de una pérdida de energía por el almacenamiento y reúso.

El $\mathrm{H}_{2}$, al ser un vector energético, también puede ser utilizado en celdas de combustible (pilas de combustible) para producir electricidad.
Recientemente el $\mathrm{H}_{2}$ ha sido utilizado como fuente alterna de energía para vehículos debido a su alto rendimiento y a que no genera contaminantes durante su uso en el auto. El auto de hidrógeno podría disminuir de manera muy considerable las emisiones de gases de efecto invernadero (GEI). Muchas compañías automotrices ya comercializan modelos impulsados por $\mathrm{H}_{2}$ (Finnerty, 2016). Por otro lado, el $\mathrm{H}_{2}$ está siendo objeto de estudio para ser utilizado como batería de los teléfonos inteligentes, pero se encuentra en fase de prueba (ARIEMA, 2003b; Cabezas et al., 2014).

\section{Inventario de las ER en México}

Las fuentes renovables son aquellas que tras ser utilizadas se pueden regenerar de forma natural - artificial en una escala de tiempo humana (SE, 2013; SENER, 2014d). El desarrollo de las ER ha sido muy lento, ya que las políticas de diversificación energética encaminadas a promoverlas no han ocupado un lugar prioritario en la planeación nacional. El desarrollo de las fuentes alternas de energía en México se destina casi en su totalidad a la generación de electricidad, utilizadas principalmente en proyectos de energización rural para satisfacer los requerimientos energéticos de comunidades aisladas y dispersas, donde la integración de la red eléctrica nacional resulta muy costosa o es prácticamente imposible (CEFP, 2001). Huacuz (2005) plantea las oportunidades que se tienen como país por el uso de ER dentro del balance energético nacional, y al mismo tiempo realiza un análisis crítico de las barreras que dificultan su explotación plena, muchas de las cuales lamentablemente aún siguen vigentes.

El país cuenta con una amplia diversidad de recursos renovables para la generación de energía, que en algunos casos es considerablemente más abundante en comparación con países con una trayectoria amplia en la explotación de estos recursos. Sin embargo, los mismos se encuentran muy dispersos en el territorio nacional. Adicionalmente, se tienen restricciones que dificultan su explotación como las características orográficas del país, la falta de infraestructura o el estatus de la región de interés en el ordenamiento territorial.

Al cierre del año 2014, la capacidad de generación vía fuentes renovables alcanzó los 16,240 MW, $25 \%$ de la capacidad de generación total. Para ese mismo año, la energía eléctrica generada vía fuentes renovables llegó a 55,003 GWh, equivalente a $18 \%$ del total generado (SENER, 2015a). La energía 
hidráulica, eólica y geotérmica, en ese orden, fueron las tres fuentes que más aportaron con $90 \%$ de la capacidad generada. A continuación se presentan brevemente los resultados por fuente de energía obtenidos de la investigación documental realizada. Para esto se consultaron documentos oficiales publicados por la SENER, la CRE y la CFE, así como trabajos de investigación científicos (Alemán Nava et al. 2014; Vidal Amaro et al., 2015).

Energía solar. La localización geográfica de México resulta ideal para el aprovechamiento de la energía solar con una radiación diaria que excede los 5 $\mathrm{kWh} / \mathrm{m}^{2}$ y llega a los $8.5 \mathrm{kWh} / \mathrm{m}^{2}$ en ciertas regiones. Estados del noroeste y norte (Sonora, Chihuahua y Baja California) presentan la mayor insolación. La energía solar puede transformarse directamente en electricidad (fotovoltaica) o calor (termosolar). El calor, a su vez, puede ser utilizado directamente para producir vapor y generar electricidad. A pesar del gran potencial de energía solar existente en el país, aún no ha sido explotado para la producción de energía eléctrica a gran escala. En este sentido, el país cuenta con 9 centrales fotovoltaicas en operación, de las cuales 7 pertenecen al sector privado y 2 a la CFE. En conjunto, la capacidad instalada es de 66,204 MW y la generación de energía es de apenas 84.13 GWh (SENER, 2013a). Se calcula que el potencial de generación de energía por esta fuente es de 6,500,000 GWh por año (PROMEXICO, 2014).

Energía eólica. La energía cinética del viento es transformada en energía mecánica mediante turbinas eólicas o aerogeneradores, que a su vez impulsan un generador eléctrico. Para 2012 se estimaba el potencial eólico del país en 71,000 MW, aunque solo se explotaba $1.7 \%$ (Alemán Nava et al., 2014). En México se tienen 26 centrales eólicas, de las cuales solo tres son operadas por la CFE. Las regiones con mejor potencial se ubican en el Istmo de Tehuantepec y en el norte de la península de Baja California. En conjunto, las centrales eólicas suman una capacidad instalada de 2,036.85 MW y una generación de energía de 6,086.28 GWh (SENER, 2013a).

Energía hidráulica. Se basa en aprovechar la energía potencial por la caída del agua desde cierta altura o por el flujo de un río o arroyo para transformarla en energía cinética mediante la rotación de turbinas a gran velocidad. Este recurso es el más ampliamente explotado en México. De acuerdo con la legislación (SEGOB, 2008) se hace distinción entre las grandes centrales (>30 MW) y las pequeñas centrales is $30 \mathrm{MW}$ ). Sinaloa, Chiapas, Michoacán y Puebla concentran el mayor número de grandes centrales, mientras que las pequeñas se encuentran ubicadas principalmente en Veracruz, Michoacán y Jalisco. Existen 79 centrales hidroeléctricas, lo que representa una capacidad instalada de 12,474.24 MW y una generación de energía de 38,820.97 GWh (SENER, 2013a).

Biomasa. También conocida como bioenergía, la cual se obtiene de la materia orgánica constitutiva de los seres vivos, sus excretas y sus restos no vivos. Los tipos de biomasa que se aprovechan en el país son: lodos de aguas residuales, residuos agropecuarios (principalmente excreta bovina y porcina), residuos industriales, residuos sólidos urbanos, bagazo de caña y licor negro. El 12\% de la energía eléctrica producida por biomasa proviene de la generación de biogas. A la fecha en México la producción de electricidad por medio de biomasa solo proviene del sector privado, suma un total de 67 centrales, con una capacidad instalada de 646.37 MW y una generación de energía de 1,399.33 GWh (SENER, 2013a).

Energía geotérmica. La energía geotérmica se obtiene mediante el aprovechamiento del calor interno de la Tierra concentrado en sistemas o yacimientos geotérmicos como manantiales termales, suelos calientes, volcanes de lodo, fumarolas, géiseres y zonas de alteración hidrotermal. México tiene un desarrollo importante en esta alternativa de generación, que lo posiciona en el cuarto lugar a nivel mundial. El estado de Baja California es considerado el de mayor potencial para la instalación de plantas generadoras de este tipo. En México hay cuatro campos geotérmicos bajo explotación: Cerro Prieto en Baja California, Las Tres Vírgenes en Baja California Sur, Los Azufres en Michoacán y Los Humeros en Puebla. Actualmente el país cuenta con 823.4 MW de capacidad instalada y una producción de 5,999.66 GWh (SENER, 2013a). Se estima un potencial de generación para este recurso de al menos 5,250 MW (Alemán Nava et al., 2014).

RESULTADOS

\section{Distribución geográfica de las ER en México}

Basados en la información anterior se logró ubicar las principales plantas de generación de energía renovable presentes en el país. Cabe señalar que solo se consideraron las plantas instaladas hasta julio de 2015, con lo que se obtuvo una representación 


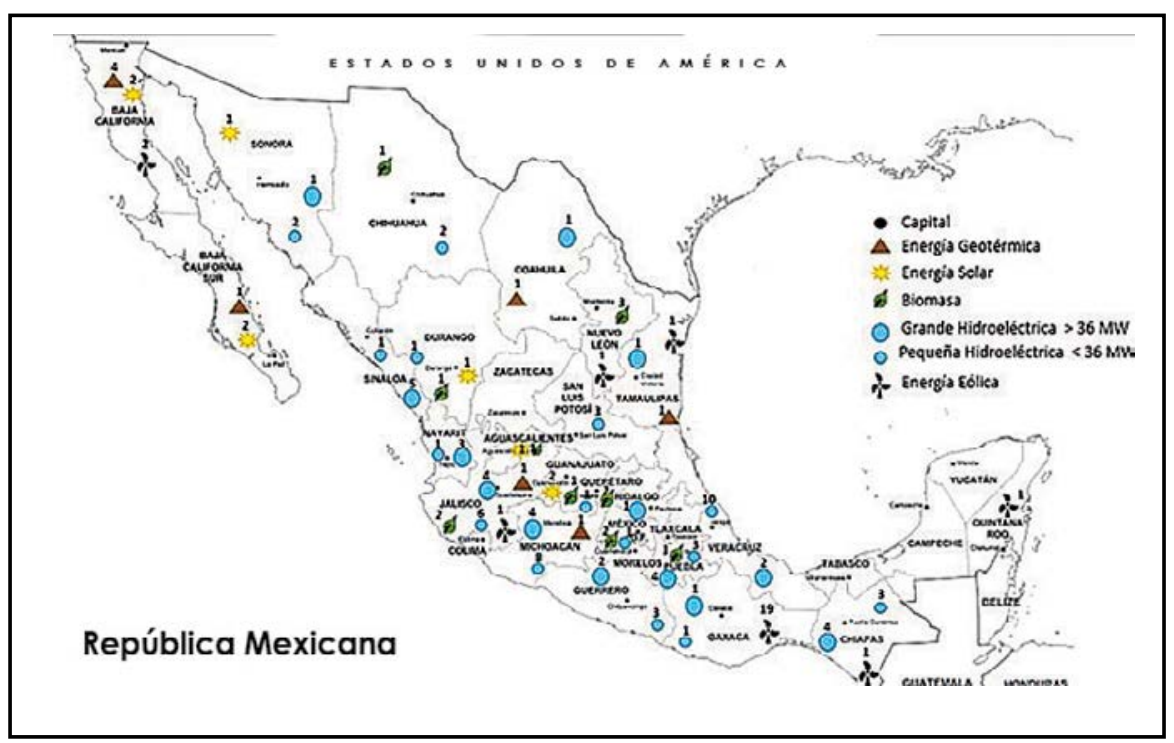

Figura 1. Ubicación de centrales de generación de energía renovable en México. Elaboración propia con información de la SENER. Julio de 2015.

gráfica del estado actual de las energías renovables, que se muestra en la Figura 1.

\section{Costos típicos de la instalación de las ER}

La principal barrera para la generación de electricidad por medio de ER y por la que la misma ha sido muy baja es el precio de la tecnología asociada. Los costos unitarios de generación de las tecnologías de energías renovables continuarán disminuyendo entre 2012 y 2035. Las principales causas de este decrecimiento serán el aumento en la implantación de las tecnologías, que acelera el progreso tecnológico, y el desarrollo de economías de escala en la fabricación de los equipos asociados (SENER, 2013b).

Generalmente la instalación de plantas de producción de energía ER suele ser muy costosa. La Figura 2 muestra los precios en promedio de la instalación de plantas por tipo de energía renovable. En dicha gráfica se aprecia que la energía eólica tiene un costo promedio menor al resto de las energías. Las hidroeléctricas, como la biomasa, tienen una gran variación debido a que el costo está muy relacionado con la capacidad de generación y la amplia variedad de tecnologías que se pueden emplear.

La razón principal es el aumento de la participación de las energías renovables en la matriz energética mundial, lo que acelerará el progreso tecnológico y el incremento de las economías de escala en la fabricación de los equipos asociados. Se espera que los costos de las tecnologías más maduras (incluida la eólica en tierra y geotérmica) tengan una caída mínima y la energía hidroeléctrica ya no tenga grandes cambios en sus costos.

\section{Perspectiva sobre la capacidad de almacenamiento de ER mediante $\mathrm{H}_{2}$}

Una vez conocida la capacidad instalada de cada una de las fuentes renovables y la importancia del $\mathrm{H}_{2}$ en la transición energética, corresponde ofrecer una perspectiva del crecimiento de las ER hasta el año 2029 considerando lo mencionado en el reporte Prospectiva de Energías Renovables 2015-2029 (SENER, 2015b). Con base en estas cifras se calcula el potencial de almacenamiento mediante el $\mathrm{H}_{2}$ como

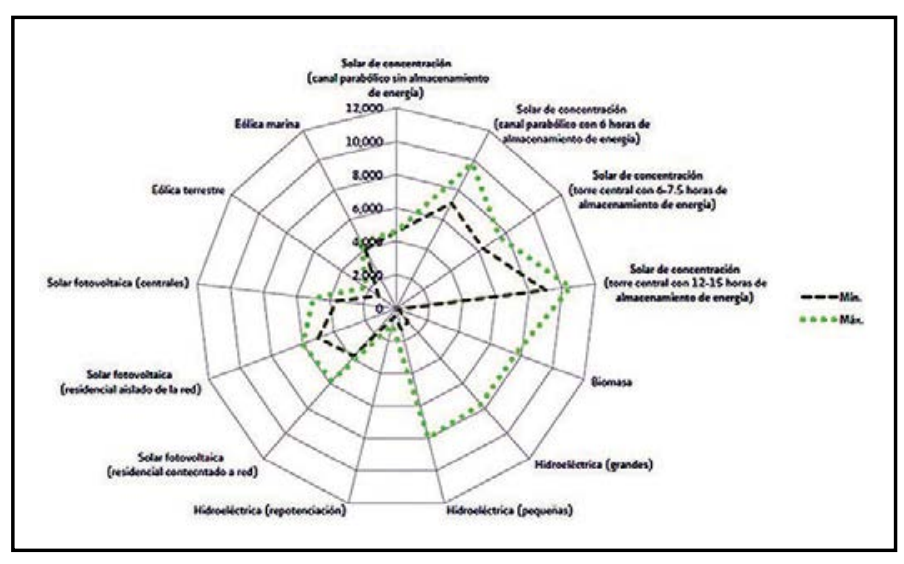

Figura 2. Aproximado de los costos de instalación de plantas de energía renovable (SENER, 2013b). 
vector energético. Esta energía almacenada podría en un futuro reconvertirse en electricidad o utilizarse como carburante en vehículos.

La Figura 3 muestra el crecimiento proyectado de las cinco fuentes mencionadas comenzando en 2015 y con incrementos en 2020, 2025 y 2029. Se indican tanto los GWh generados por año y tipo de ER como el porcentaje que representan en el balance de ER a través del tiempo. Se espera un incremento mayor para la energía eólica seguida con desarrollos importantes para la geotermia y la energía solar, para las instalaciones de biomasa se estima un aumento moderado (SENER, 2015b). Para la estimación de los escenarios se considera que no se abrirán plantas adicionales a las estimadas en el reporte de la SENER (2015b) durante el periodo evaluado.

En la Figura 3 también se aprecia el potencial anual de producción de hidrogeno, en miles de toneladas de $\mathrm{H}_{2}$, que podría obtenerse a partir de la producción de electricidad de las ER. La generación de hidrógeno se estima considerando el proceso de la electrólisis de agua. En este proceso, la molécula de agua se descompone en hidrógeno y oxígeno con ayuda de una corriente eléctrica (para el escenario explorado en este trabajo se usa la electricidad generada por las ER).

Existen diversas tecnologías para llevar a cabo la electrólisis. En este análisis se asume el uso de la tecnología de electrólisis con membrana de intercambio protónico (PEM, por sus siglas en inglés: polymer electrolyte membrane). El consumo de energía demandado por la tecnología PEM es de $55 \mathrm{kWh}$ por $\mathrm{kg}$ de $\mathrm{H}_{2}$ producido, que equivale aproximadamente a $75 \%$ de eficiencia (Dagdougui, 2012). Sin embargo, un kilogramo de hidrógeno sólo puede aportar unos $33 \mathrm{kWh}$ de energía cuando es empleado. Este tipo de pérdidas existen en el uso de todos los productos que pasan por diferentes etapas. El valor de la gráfica fue obtenido al dividir el total de ER entre los $55 \mathrm{kWh}$ necesarios para producir un $\mathrm{kg} \mathrm{de \textrm {H } _ { 2 }}$ y posteriormente el valor se multiplica por el factor 33 kWh de energía que se puede aprovechar, tal como se muestra en la tesis de De León Almaraz (2014). Por ejemplo, si en el año 2029 se tiene una capacidad de producción de 118,560 GWh/año, la misma se convertirá en 71,135 GWh/año si previamente fue almacenada en forma de hidrógeno.

De acuerdo con los datos obtenidos, el potencial para el año 2029 (Figura 3) es de una producción de

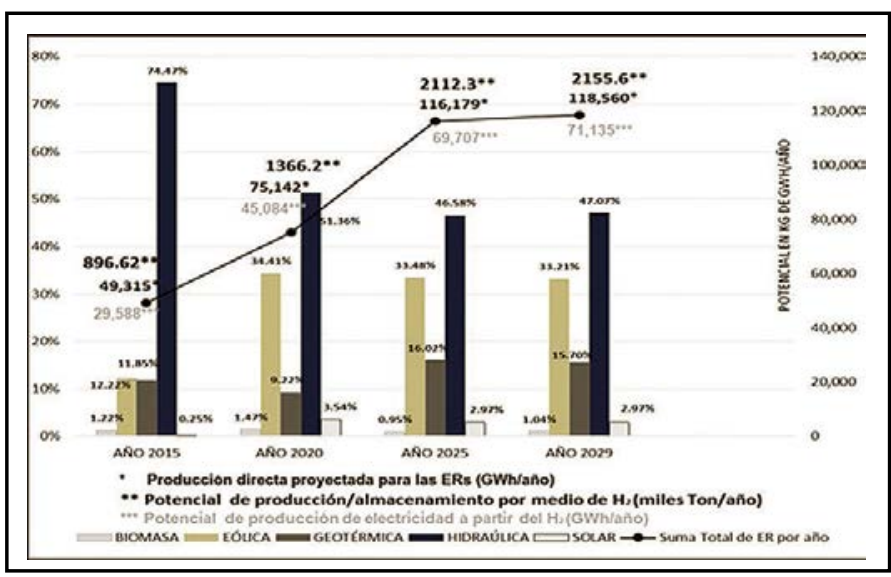

Figura 3. Perspectivas de energía renovable en México hasta el año 2029. Se representa la participación de cada fuente en el periodo evaluado y el potencial acumulado en $\mathrm{kg} \mathrm{de} \mathrm{H}_{2}$. Elaboración propia con datos proporcionados por la SENER (2015b).

$2,155,000$ † de $\mathrm{H}_{2}$ por año, basado en el uso total de ER, equivalente a 118,560 GWh por año.

Las energías renovables acopladas al hidrógeno son una vía prometedora para la descarbonización de los sistemas de energía a nivel mundial. La consideración del hidrógeno como vector energético ofrece soluciones eficaces para el control de las emisiones, así como para el aseguramiento y el abastecimiento energético limpio. Algunos países como Inglaterra, Alemania, Estados Unidos, España, Francia, Dinamarca, Rusia, Japón, entre otros, se han dedicado a estudiar los múltiples beneficios que ofrecen desde un punto de vista económico, social y ambiental; por lo que sin duda una nueva era energética es inminente y hay que estar preparados para afrontarla de forma adecuada.

Se discute mucho acerca de la preferencia de utilizar la electricidad generada por medio de las energías renovables de manera directa y ésta es sin duda la mejor opción; sin embargo, para el caso de las ER se requeriría una enorme inversión en instalaciones de líneas de alta tensión teniendo en cuenta el problema de las altas variaciones de generación por la intermitencia. Por ejemplo, si un día la capacidad de producción por medio de una estación de turbinas eólicas es superior a la capacidad de transferencia de las mismas o a la demanda de electricidad, esta energía se desperdicia si no es almacenada por algún medio como el hidrógeno; si por otro lado, existe un periodo en el cual las condiciones meteorológicas no son adecuadas, se deja de producir, teniendo un grave riesgo de no abastecer la demanda. Por 
supuesto que las proyecciones estimadas por la SENER parten de la base histórica de capacidades de producción de las ER; sin embargo, la capacidad de producción puede ser aumentada mediante el uso de $\mathrm{H}_{2}$ creando regiones con autonomía energética, reduciendo el transporte y con la gran ventaja de que el $\mathrm{H}_{2}$ puede ser utilizado como carburante en automóviles sin emisiones en su uso y generación muy baja de emisiones si es producido a partir de renovables.

Con el nuevo marco legal de la Reforma Energética se hace más flexible la participación del sector privado en la generación y comercialización de energía eléctrica. Sin embargo, el Estado conservará la planeación y control del Sistema Eléctrico Nacional, así como las actividades relativas a la transmisión y distribución de energía eléctrica (SENER, 2014C). Esta reforma prevé y promueve el fortalecimiento de las ER apoyada por la Ley para el Aprovechamiento de las Energías Renovables y el Financiamiento de la Transición Energética (LEARFTE), así como por el Programa Especial para el Aprovechamiento de Energías Renovables (PEAER), en donde se indica una participación de $22.81 \%$ de las energías limpias en la generación para 2018 hasta alcanzar $26.49 \%$ para 2027 (SENER, 2014b). Estudios de factibilidad deberían ser realizados para tener una perspectiva más clara con respecto al hidrógeno como opción de almacenamiento de las ER.

Resulta indispensable empezar a observar la forma en que otros países han resuelto los proble- mas inherentes del sector ER-hidrógeno, como lo es el almacenamiento y distribución, ya que la falta de infraestructura es uno de los principales inconvenientes que enfrenta actualmente la economía del hidrógeno. Es necesario realizar un estudio detallado de la cadena de suministro del hidrógeno a través de diferentes fuentes de energía para abastecer diversos nichos de mercado. Finalmente, el desarrollo de las ER y el hidrógeno se proyecta como un polo de desarrollo y crecimiento en los años por venir al poder resultar en la creación de un gran número de fuentes de empleo directo.

CONCLUSIONES

El sector de ER, a pesar de ser una industria naciente en México, ha crecido favorablemente en los últimos años. El mercado mexicano es amplio y atractivo sobre todo por el potencial solar, eólico y geotérmico. Los compromisos adquiridos por el país sobre reducción de emisiones de $\mathrm{CO}_{2}$ hacen imperativa la pronta transición energética, ya que se espera que para 2024 más de 30\% de la generación de electricidad provenga de energías limpias. Actualmente la energía hidráulica es la fuente más ampliamente explotada y continuará siéndolo en los próximos años. Este panorama motiva a la reflexión sobre el problema de intermitencia y de uso de la red eléctrica para la satisfacción de la demanda de electricidad que provocará el desarrollo de este sector, lo que hace interesante el estudio del posible establecimiento de empresas generadoras de $\mathrm{H}_{2}$ como vector energético.

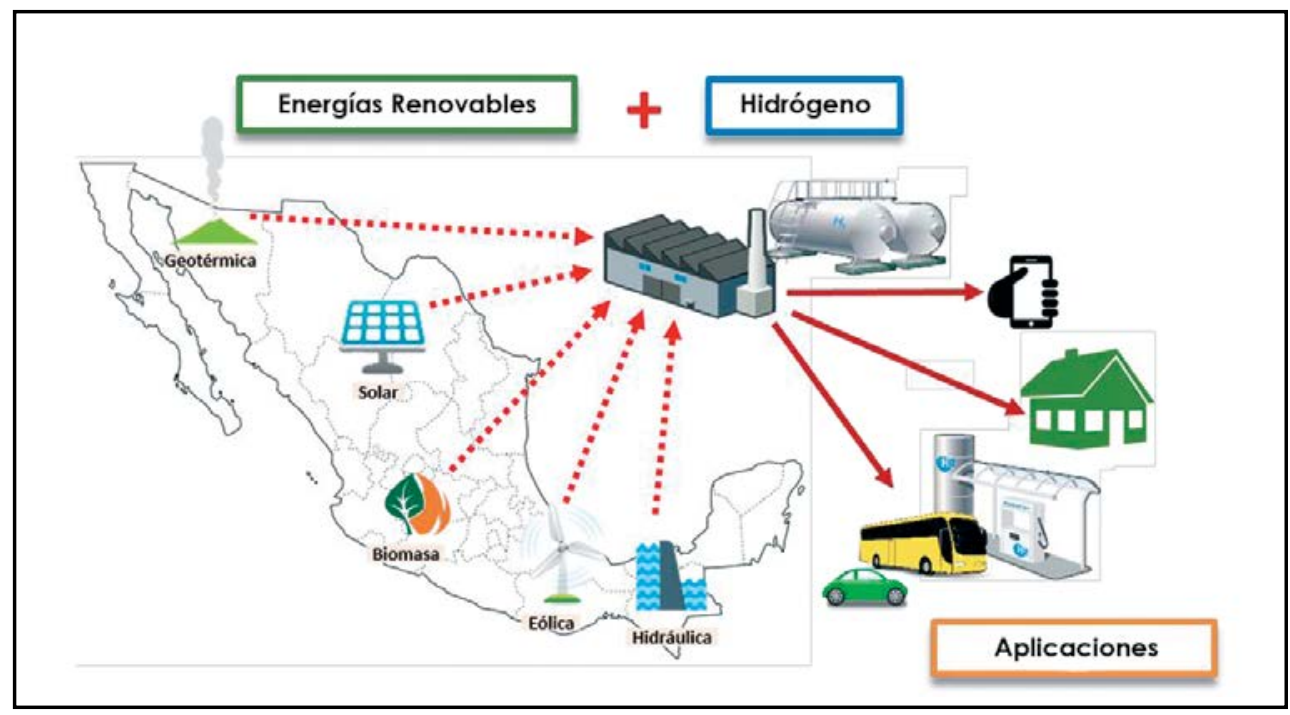

Figura 4. El hidrógeno en conjunto con las ER favorece la transición energética del país al ofrecer una solución a la intermitencia de las ER y poder ser utilizada en varias aplicaciones de forma sustentable. Elaboración propia. 
IIVESTIGACIÓn Y CIERGIA DE LA UחIVERSIDAD AUTÓnOMH DE RGUASCALIERTES
El objetivo de este trabajo fue realizar una estimación del potencial de la utilización de ER para la producción de $\mathrm{H}_{2}$ para resolver el problema de la intermitencia de las ER.

De acuerdo con la estimación prospectiva que se llevó a cabo en este trabajo, se puede concluir que el potencial de almacenamiento de $\mathrm{H}_{2}$ es atractivo, ya que podría lograrse un abastecimiento de 118,560 GWh por año de energía renovable.

Por el momento se han establecido los primeros escenarios que serán utilizados como base para estudiar la viabilidad del uso del $\mathrm{H}_{2}$ como nuevo vector energético, y de esta forma plantear la posible configuración de su cadena de abastecimiento en México.

\section{Agradecimientos}

Se reconoce y agradece ampliamente al programa Interinstitucional para el Fortalecimiento de la Investigación y el Posgrado del Pacífico (DELFIN)-CONACYT por el financiamiento para realizar la presente investigación, así como al Centro de Investigación en Matemáticas, A. C. (CIMAT) unidad Aguascalientes por su apoyo y las facilidades prestadas.

LITERATURA CITADA

- $\quad$ ALEMÁN NAVA, G.S. et al. Renewable energy research progress in Mexico: a review. Renewable and Sustainable Energy Reviews, 32, 140-153, 2014.

- $\quad$ BALL, M. y WIETSCHEL, M. The future of hydrogen-opportunities and challenges. International Journal of Hydrogen Energy, 34 (2): 615-627, 2009.

- BHATTACHARYA, $M$. et al. The effect of renewable energy consumption on economic growth: evidence from top 38 countries. Applied Energy, 162, 733-741, 2016.

- BUYUKSAHIN, B. Speculation demystified: virtuous volatility. The Journal of the International Energy Agency, 34-35, 2012.

- CABEZAS, M. D. et al. Hydrogen energy vector: demonstration pilot plant with minimal peripheral equipment. International Journal of Hydrogen Energy, 39(32): 18165-18172, 2014.

- DAGDOUGUI, H. Decision support systems for sustainable renewable energy systems and hydrogen logistics : modelling, control and risk analysis. PhD thesis. France: École Nationale Supérieure des Mines de Paris-Italy: Universita degli studi di Genova, 2012

- DELEÓN ALMARAZ, S. Multi-objective optimisation of a hydrogen supply chain. PhD thesis. France: Université de Toulouse, 2014.

- GAlarzA, C. Hidrógeno. D. F., México: Comisión Nacional Para El Uso Eficiente de La Energía, 2008.

- HUACUZ, J. M. The road to green power in Mexico-reflections on the prospects for the large-scale and sustainable implementation of renewable energy. Energy Policy, 33(16): 2087-2099, 2005

- IEA (INTERNATIONAL ENERGY AGENCY). Energy and climate change. France: OECD-IEA, 2015.
- IEA (INTERNATIONAL ENERGy AGENCY). Renewable Energy. Medium-Term Market Report 2014. Market Analysis and Forecasts to 2020. Executive Summary. France: OECD-IEA, 2014.

- IEA (INTERNATIONAL ENERGY AGENCY). World Energy Outlook 2009. France: OECD-IEA, 2009.

- IPCC CORE WRITING TEAM et al. Climate Change 2007. Synthesis Report. Contribution of Working Groups I, II and III to the Fourth Assessment Report of the Intergovernmental Panel on Climate Change. Geneva, Switzerland: IPCC, 104 pp., 2007.

- SALAMEH, Z. Renewable energy system design. US: Academic Press-Elsevier, 2014.

- SCHOLTEN, D. y BOSMAN, R. The geopolitics of renewables; exploring the political implications of renewable energy systems. Technological Forecasting and Social Change, 103, 273-283, 2016.

- SE (SECRETARÍA DE ECONOMÍA). Energías Renovables. México: Autor, 2013.

- SEGOB (SECRETARÍA DE GOBERNACIÓN). Decreto por el que se expide la Ley para el Aprovechamiento de las Energías Renovables y el Financiamiento de la Transición Energética. Diario Oficial de la Federación, 28 de noviembre de 2008.

- SENER (SECRETARÍA DE ENERGÍA). Balance Nacional de Energía 2013. México: Autor, 2014a.

- SENER (SECRETARÍA DE ENERGÍA). Informe Sobre La Participación de Las Energías Renovables En La Generación de Electricidad En México Al 31 de Diciembre de 2014. México: Autor, 2015a.

- SENER (SECRETARÍA DE ENERGÍA). Inventario Nacional de Energías Renovables. México: Autor, 2013a. 
IIVESTIGAGIÓO Y CIERCIA DE LA UחIVERSIDAD AUTÓNOMA DE RGUASCALIERTES

101

- Sener (SECRETARía de ENERgíA). Programa Especial Para el Aprovechamiento de Energías Renovables. México: Autor, $2014 b$.

- Sener (SECRETARÍA DE ENERGía). Prospectiva de Energías Renovables 2013-2027. México: Autor, 2013b.

- SENER (SECRETARÍA DE ENERGÍA). Prospectiva de Energías Renovables 2015-2029. México: Autor, 2015b.

- SENER (SECRETARÍA DE ENERGÍA). Prospectiva de Gas Natural y Gas L.P. 2015-2029. México: Autor, 2015c.

- Sener (SECRETARÍA DE enERGÍA). Prospectiva del Sector Eléctrico 2014-2028. México: Autor, 2014c.

- Sener (SECRETARÍA DE energía). Prospectiva del Sector Eléctrico 2015-2029. México: Autor, 2015d.

- SENER (SECRETARÍA DE ENERGÍA). Recursos Renovables Para La Producción de Energía En México. Mexico: Autor, 2014d.

- VIDAL AMARO, J. J. et al. Optimal energy mix for transitioning from fossil fuels to renewable energy sources-the case of the mexican electricity system. Applied Energy, 150, 80-96, 2015.

\section{De páginas electrónicas}

- AIR LIQUIDE. Air Liquide et l'hydrogène. France: Air Liquide, 2008. Recuperado de http://www.planete-hydrogene.com/fr/ air-liquide-et-lhydrogene.html

- ARIEMA Energía y Medio Ambiente SL. Producción de H2 $>$ pilasde.combustible. España: ARIEMA Energía y Medio Ambiente SL., 2003a. Recuperado el 11 de octubre de 2015, de http://www.pilasde.com/tecnologia-del-hidrogeno/ produccion-h2

- $\quad$ ARIEMA Energía y Medio Ambiente SL. Usos Del Hidrógeno $>$ pilasde.combustible. España: ARIEMA Energía y Medio Ambiente SL., 2003b. Recuperado el 11 de octubre de 2015, de http://www.pilasde.com/tecnologia-del-hidrogeno/usos-h2

- CADET, G. La question énergétique au mexique: où en est la réforme de l'électricité? Observatoire des Amériques. Montreal, Quebec, Canadá: CEIM-UQAM, 2003. Recuperado el 22 de agosto de 2016, de http://dspace.africaportal. org/jspui/bitstream/1 23456789/387/1/La\%20question\%20 energetique\%20au\%20Mexique\%20-\%200u\%20en\%20est\%20 la\%20reforme\%20de\%20lelectricite.pdf? 1

- cámara de diputados-CefP (Centro de Estudios de las Finanzas Públicas). Evolución y perspectiva del sector energético en México, 1970-2000. México: CEFP, 207 pp., 2001.
Recuperado el 19 de septiembre de 2016, de http://www. cefp.gob.mx/intr/edocumentos/pdf/cefp/cefp0512001.pdf

- ENERGÍA y SOCIEDAD. Cambio climático. España: Energía y sociedad, 2014. Recuperado el 11 de octubre de 2015, de http://www.energiaysociedad.es/ficha/cambio-climatico

- FERNÁNDEZ ORDÓÑEZ, M. Intermitencia renovable: otro de los problemas. Blogs Madrid Más > Ciencia y tecnología, 8 de abril de 2010. Recuperado de http://www.madrimasd.org/blogs/ ciencianuclear/2010/04/08/131696

- FINNERTY, J. Hydrogen cars: new gouvernment funding for fuel cell vehicles. Autoexpress, 10 de mayo de 2016, 5:00 p.m. Recuperado el 15 Septiembre de 2016, de http://www. autoexpress.co.uk/car-news/93180/hydrogen-cars-newgovernment-funding-for-fuel-cell-vehicles

- KOTTASOVA, I. Oil's wild ride isn't over yet. CNN Money. Market Movers and Shakers, 13 de octubre de 2015, 12:13 p.m. Recuperado el 8 de noviembre de 2015, de http://money.cnn. com/2015/10/13/news/economy/oil-prices-iea-volatility/

- MÉXICO NEWS NETWORK. Impulsa Peña Nieto en COP21 iniciativa para fijar precio a carbono. Mexico News Network, martes 1 de diciembre de 2015, 13:52. Recuperado el 2 de diciembre de 2015, de http://www.mexiconewsnetwork.com/ es/nacional/impulsa-epn-cop21-precio-carbono/

- NOW. NATIONAL ORGANISATION HYDROGEN AND FUEL CELL TECHNOLOGY [Portal electrónico]. Berlín, Alemania: NOW $\mathrm{GmbH}, 2012$. Recuperado el 1 de diciembre de 2015, de https://www.now-gmbh.de/en

- PRESIDENCIA DE LA REPÚBliCA. Declaración de Líderes de América del Norte sobre la Alianza del clima, energía limpia y medio ambiente. México: Presidencia de la República, 2016. Recuperado el 22 de septiembre de 2016, de https://www. gob.mx/presidencia/documentos/declaracion-de-lideres-deamerica-del-norte-sobre-la-alianza-del-clima-energia-limpia-ymedio-ambiente

- PROMÉXICO TRADE AND INVESTMENT. Negocios. The new mexican energy model. México: ProMéxico, 2014. Recuperado de http://www.promexico.gob.mx/documentos/revistanegocios/html/2014-11/english/11-2014/art01.html

- SCANDINAVIAN HYDROGEN HIGHWAY PARTNERSHIP. [Portal electrónico]. Gotemburgo, Suecia: Scandinavian Hydrogen Highway Partnership, 2011. Recuperado el 1 de diciembre de 2015, de http://www.scandinavianhydrogen.org

- UN (UNITED NATIONS). United Nations Conference on Climate Change-COP21. 2015. Recuperado el 1 de diciembre de 2015, de http://www.cop21.gouv.fr/en/learn/what-is-cop21 